\title{
Analysis of Energy Efficiency of a Test Rig for Air Springs
}

\author{
Ivan Okorn* - Marko Nagode \\ University of Ljubljana, Faculty of Mechanical Engineering, Slovenia
}

During dynamic testing of vehicle air springs, work is required for loading the springs. This work is partially returned by the spring to the driving system during the release phase. Testing is energy-efficient when at least a portion of the returned work can be utilised. This was taken into account in the design of the new inovative test rig for the simultaneous testing of four air springs. There is a phase shift between the phases of loading individual air springs; thus, the work returned to the drive system by a spring during its release is also used for loading another spring. The test rig was constructed and operates in the laboratory of an air spring manufacturer. We developed a computer program to analyse the energy situation in the test rig. The program calculates the work required for loading the springs, power and friction for different sizes of springs and test conditions. In this paper, computational algorithms are deduced and the results of the calculation for the treated spring are presented. The energy situation in the test rig during start-up and operation is discussed, taking into account the energy loss due to the hysteresis of springs and friction losses. The friction losses are evaluated for different implementations of critical elements. The influence of a flywheel on conditions during the start-up and operation of the test rig is analysed.

Keywords: air spring, test rig, energy efficiency, spring hysteresis, friction losses

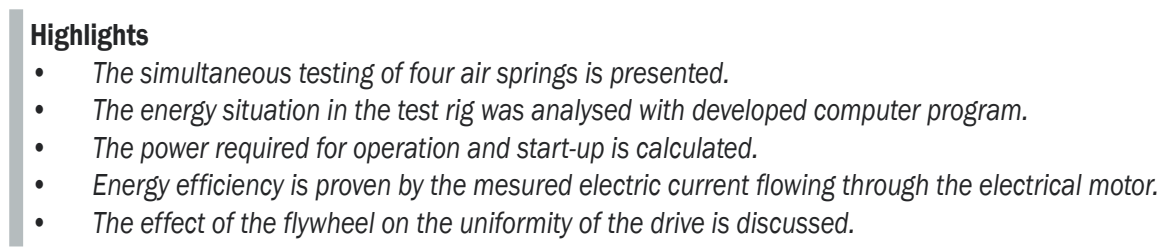

\section{INTRODUCTION}

Dedicated test rigs are used for the dynamic testing of air springs. These test rigs allow a change of geometry of fixing the springs, which reflects the real spring fixing in a vehicle. Our test rig was constructed for a well-known manufacturer of air springs. It is primarily used for type-approval testing in order to confirm the customer and legislative requirements. The users of springs, who install the springs to their vehicles, define the amplitude and frequency of load, and the minimum number of cycles until the critical damage may appear in a spring. The test rig is also used for research. The paper [1] presents a method for estimating the fatigue life of the air-spring. Experimental data were obtained from several accelerated experiments using constant load amplitude. In [2], the influence of the cord angle on the air-spring fatigue life is presented. Constant amplitude tests were used to make life predictions based on the standardised load spectrum in [3].

Test rigs used for testing air springs can be divided into two major groups: hydraulic and mechanical test rigs. Usually, only one spring is tested at a time on hydraulic test rigs. The work returned by the spring during unloading remains unused. During the test, it is possible to modify the amplitude and frequency of load, which is not possible in mechanical test rigs.
Mechanical test rigs are used mainly for type-approval tests. The amplitude and frequency of the sinusoidal load are set at the start of the test, and they remain the same throughout the test. On the existing mechanical test rigs, two air springs are tested at the same time, being alternatively loaded and released. The new test rig allows the simultaneous testing of four springs. All four springs are loaded under the same conditions (same amplitude and frequency); only the loading is done with a phase shift. During the test, a large part of the work returned by the spring during its release is used for loading the adjacent spring. The energy efficiency of the test rig will be analysed in the following section. The hysteresis of a spring and friction losses in the mechanical elements will also be taken into account.

We developed a computer program that calculates the load of the test rig components and friction losses during the test of any spring. The program calculates the power for start-up and testing. Calculation results for a spring that was tested on the new test rig will be presented. We used a flywheel to ensure the uniformity of the drive. The mass moment of the inertia of the flywheel was determined on the basis of surpluses and deficits of work with respect to the medium work for a load cycle. 


\section{MOUNTING AND LOADING THE AIR SPRING}

When designing the test rig, we took into account the dimensions of the spring and the method of mounting the spring to the vehicle. Methods are presented in [4] and [5]. An example of mounting is shown in Fig. 1.

A lever is fixed on the vehicle's axle and connected to the chassis of the vehicle via a pivot. An air spring is mounted between the lever and the chassis. The spring can be mounted in front of the axle as shown in Fig. 1, directly below the axle or behind the axle of the vehicle.

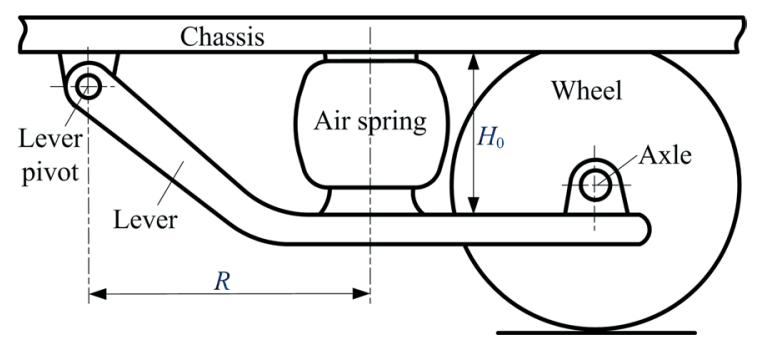

Fig. 1. Mounting the air springs on a vehicle

Fig. 2 schematically shows the mounting of springs on the test rig. One side of the spring is fixed on an inclined plate on the lever, and the other side on an inclined plate on the test rig framework. The two inclined plates are parallel in the horizontal position of the lever. Eccentric with a rotation point 3 pushes the lever in point 4 via the slider. The lever oscillates around point 5 , loading one spring and releasing the other one via inclined plates.
Two pairs of springs are loaded on the test rig, each pair with its own lever and its corresponding eccentric. In this paper, we will use the terms 'upper' and 'lower' spring, 'left' and 'right' eccentric. The springs are alternately loaded and released during rotation of the eccentrics. While the upper spring is released on the right lever, the lower spring is loaded on the left lever. The spring that is released returns the work to the system, while the spring that is compressed uses the work from the system. The two eccentrics can be rotated to each other by any angle. The shift on an active test rigs is $90^{\circ}$.

With the test rig, it is possible to continuously adjust the distance between the lever rotation point and the centre of the spring $R$, the eccentricity of the slider $r$, the inclination of the spring, the distance between the inclined plates and the frequency of loading the springs. When setting the distance $R$, the rotation point of the lever 5 is moved, both with respect to the test rig framework and lever. Friction joints with pre-stressed screws are used for mounting the lever to the pivot axle and for mounting the pivot housing to the framework. When the pivot position is changed, the lever is fixed with respect to the test rig framework.

The test rig drive is shown schematically in Fig. 3. A three-phase asynchronous electric motor with a power of $P_{1}=11 \mathrm{~kW}$ and a rotational speed of $n_{1}=1450 \mathrm{rpm}$ drives the shaft 1 , on which the flywheel is mounted. The power is transmitted from the shaft 1 to 3 by two belt drives. Eccentrics that

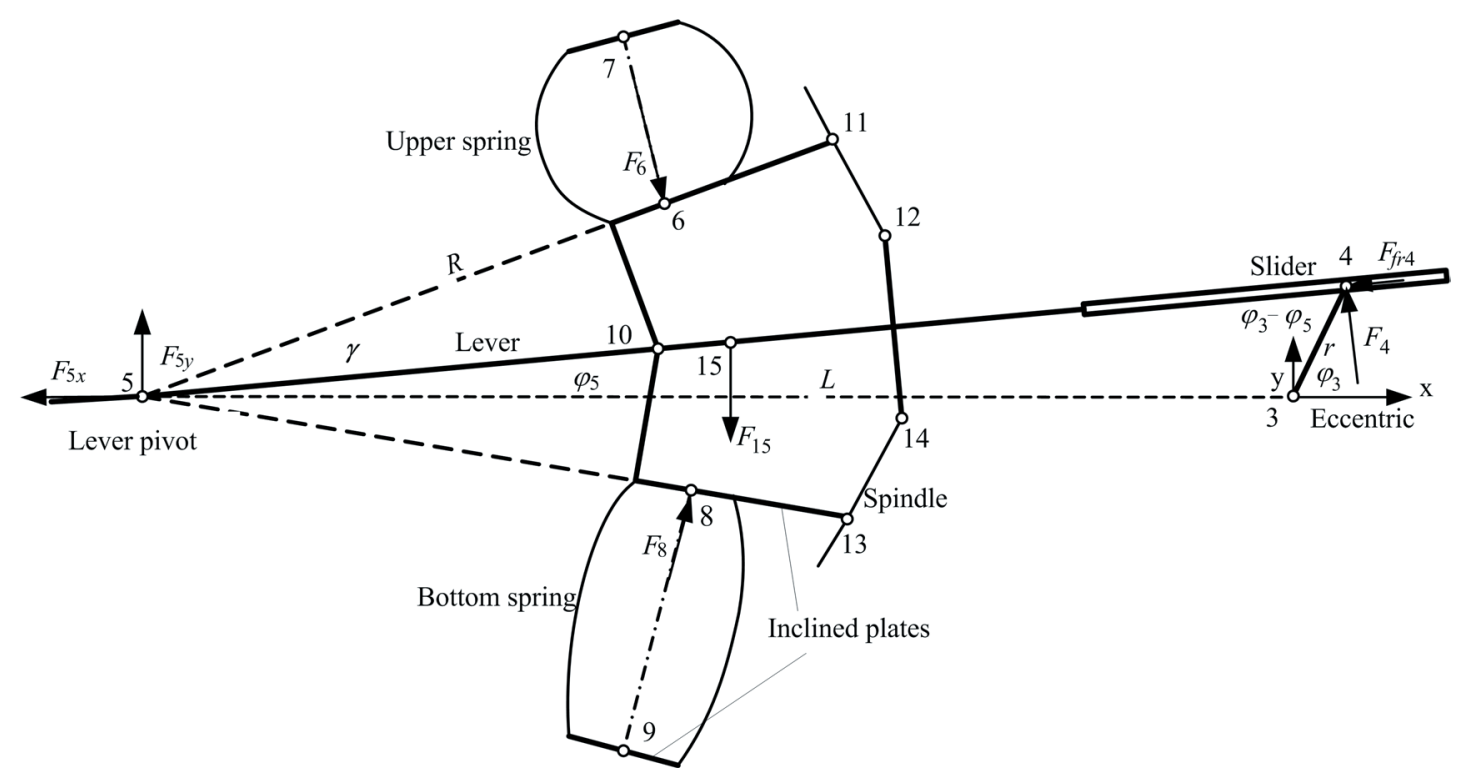

Fig. 2. Loading the air spring on the test 
push levers are mounted at both ends of the shaft 3 . The frequency regulation of the electric motor allows setting the frequency of loading the springs between 0.5 and $3.5 \mathrm{~Hz}$.

The test rig consists of three functional components: mechanical part with a drive, pneumatic part and controller. This article discusses only the mechanical part in detail. The display controller displays air pressure in all four springs, the electric motor current, the rotational speed of shaft 3 (i.e. the testing frequency) and the number of load cycles.

The first indication of a damaged spring is a pressure drop in the spring. The average pressure must be the same in all four springs. The average pressure and the pressure drop when the test rig automatically stops are set on the controller. By monitoring the pressure on the controller screen, the changing working force of the air spring can be monitored indirectly.

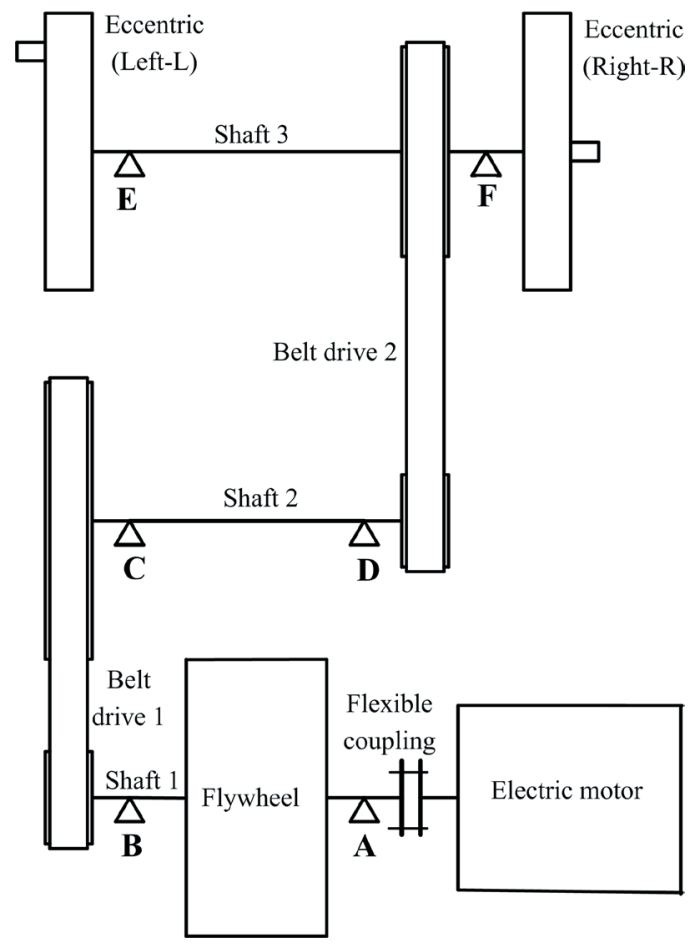

Fig. 3. Outline of the test rig drive

The test rig has built-in high-current protection. It stops automatically if the operating current exceeds the velue $60 \%$ of rated current.

\section{LOAD ON THE ECCENTRIC}

Forces in a particular eccentric depend on the work force of the spring, and the weight and inertia of the lever. We shall deal with the load on the right eccentric. The load on the left eccentric is the same; it just occurs with a phase shift. Variables related to the left eccentric are labelled as ' $L$ ', while variables for the right eccentric are labelled as ' $R$ '. The height of the springs in the horizontal position of the lever is known as installation height and denoted by ' $H_{0}$ '.

\subsection{Work Force of a Spring}

The program calculates the work forces in springs $F_{6}$ and $F_{8}$ on the basis of the current height of springs and the known characteristics of springs. Geometrical parameters of the lever and characteristic points on it are presented in Fig. 2. The positions of the points are described by vectors with their origin in the rotation point of the eccentric. The vectors that define anchorages of springs depend on the mounting parameters $R, L$ and $\gamma$, the mounting height of springs $H_{0}$ and the angle of rotation of the lever $\varphi_{5}$.

$$
\begin{gathered}
\vec{r}_{6}=\left(\begin{array}{l}
R \cos \left(\varphi_{5}+\gamma\right)-L \\
R \sin \left(\varphi_{5}+\gamma\right)
\end{array}\right), \\
\vec{r}_{7}=\left(\begin{array}{l}
R \cos \gamma-H_{0} \sin \gamma-L \\
R \sin \gamma+H_{0} \cos \gamma
\end{array}\right) .
\end{gathered}
$$

The lever's angle of rotation changes during the test; it is calculated using the following equation:

$$
\varphi_{5}=\arctan \frac{r \sin \varphi_{3}}{L+r \cos \varphi_{3}} .
$$

The heights of springs at a defined angle of rotation equal the absolute difference of vectors. For the difference of vectors:

$$
\vec{r}_{7}-\vec{r}_{6}=\left(\begin{array}{c}
R\left(\cos \gamma-\cos \left(\gamma+\varphi_{5}\right)\right)-H_{0} \sin \gamma \\
R\left(\sin \gamma-\sin \left(\gamma+\varphi_{5}\right)\right)+H_{0} \cos \gamma
\end{array}\right),
$$

the absolute value is

$$
\begin{gathered}
H=\left|\vec{r}_{7}-\vec{r}_{6}\right|= \\
=\sqrt{2 R^{2}\left(1-\cos \varphi_{5}\right)+H_{0}\left(H_{0}-2 R \sin \varphi_{5}\right)} .
\end{gathered}
$$

An air spring characteristic depends on the type and dimension of the spring, and air pressure in the spring [6]. The influance of the rubber bumpers built into the air-springs is analysed in [7]. Dynamic load-sharing analysis of longitudinal-connected air suspensions is presented in [8]. The pressure in the spring changes during contraction and expansion of 
the spring, causing changes in the work force of the spring.

The measured characteristic of the treated air spring is shown in Fig. 4. The installation height of the spring in the horizontal position of the lever is $H_{0}=254 \mathrm{~mm}$. We used the fourth order polynomials to define the load and release curves. Approximately $30 \mathrm{~kW}$ (maximal) is required in hydraulic test rig for loading one spring with a frequency of $3.5 \mathrm{~Hz}$. Losses in the hydraulic system are not included in this value. The load work was determined on the basis of the measured characteristics of the spring (Fig. 4).

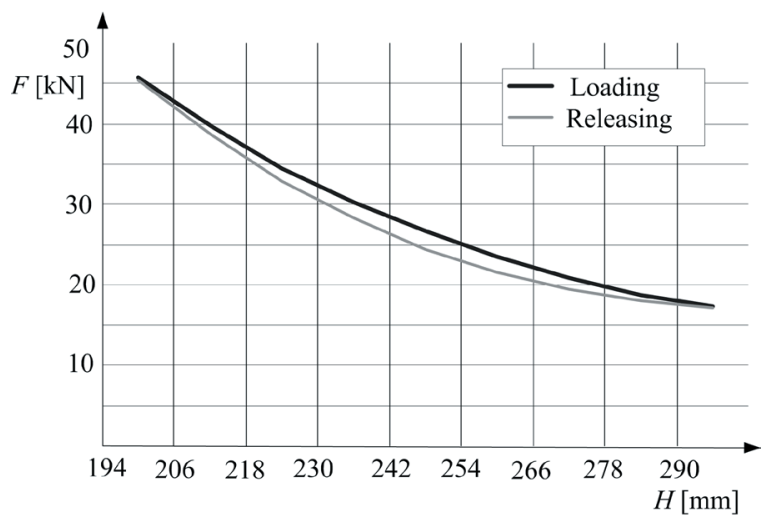

Fig. 4. Measured characteristics of the treated spring

\subsection{Acceleration of the Lever and Force of Inertia}

Acceleration of the lever is calculated by deriving Eq. (3) for rotation of the lever twice.

$$
\ddot{\varphi}_{5}=\frac{r L \sin \varphi_{3}\left(r^{2}-L^{2}\right)}{\left(L+r \cos \varphi_{3}\right)^{4}} \cdot \dot{\varphi}_{3}^{2}+\frac{r\left(r+L \cos \varphi_{3}\right)}{\left(L+r \cos \varphi_{3}\right)^{2}} \cdot \ddot{\varphi}_{3} .
$$

Torque becouse of inertia with respect to the rotation point of the lever:

$$
M_{t i}=J_{5} \cdot \ddot{\varphi}_{5}=\left(J_{15}+m_{15} \cdot l_{5,15}^{2}\right) \cdot \ddot{\varphi}_{5} .
$$

The moment of inertia of the lever with respect to its rotation point $J_{5}$ depends on the moment of inertia $J_{15}$ around the centre of mass, mass of the lever $m_{15}$ and the distance between the rotation point and the centre of mass $l_{5,15}$. The mass of the lever together with two tilting plates is $m_{15}=420 \mathrm{~kg}$ and the centre of mass moment of inertia is $J_{15}=225 \mathrm{kgm}^{2}$. The flywheel is installed into the drive system, so the variation of the angular velocity of the eccentric is negligible. The results of the calculations indicate a negligible influence of the second term in Eq. (6).

\subsection{Normal Force on the Slider}

The slider of the eccentric presses on the guide with a force $F_{4}$, which is perpendicular to the lever. The losses due to friction and thus heating of the slider depend on the size of the force. We have derived the term for a normal force on the eccentric from the moment equilibrium equation for the rotation point of a lever. We assumed that the forces of the springs act perpendicular to the inclined plates of the lever.

$$
\begin{gathered}
\sum M_{i 5}=0, \\
F_{8} \cdot R-F_{6} \cdot R-F_{15} \cdot l_{5,15} \cdot \cos \varphi_{5}+ \\
+F_{4} \cdot\left(L+r \cos \left(\varphi_{3}+\varphi_{5}\right)\right)-J_{5} \cdot \ddot{\varphi}_{5}=0, \\
F_{4}\left(\varphi_{3}\right)=\frac{-\left(F_{8} \cdot R-F_{6} \cdot R\right)}{L+r \cos \left(\varphi_{3}+\varphi_{5}\right)}+ \\
+\frac{F_{15} \cdot l_{5,15} \cdot \cos \varphi_{5}}{L+r \cos \left(\varphi_{3}+\varphi_{5}\right)}+\frac{J_{5} \cdot \ddot{\varphi}_{5}}{L+r \cos \left(\varphi_{3}+\varphi_{5}\right)} .
\end{gathered}
$$

The first term takes into account the spring forces, the second term the lever's own weight, and the third one the influence of inertia of the lever. Analyses have shown that the inertia of the lever has a significant impact on the load of the slider. We performed special analyses in which we took into account only the weight and inertia of the lever. Work forces of the spring are negligible in the start-up phase because of minimal air pressure in the springs. Only when the shafts rotate at the testing frequency does the pressure in the springs begin to increase gradually to the nominal pressure.

\subsection{Circumferential Force on the Eccentric Crank}

The circumferential force on the eccentric crank depends on the normal force $F_{4}$ and friction force on the slider $F_{f r 4}$. It is calculated with the following equation:

$$
\begin{aligned}
& F_{t 4 R}\left(\varphi_{3}\right)=F_{4}\left(\varphi_{3}\right) \cdot \cos \left(\varphi_{3}-\varphi_{5}\right)+ \\
& +\left|F_{f r 4}\left(\varphi_{3}\right) \cdot \cos \left(\frac{\pi}{2}-\left(\varphi_{3}-\varphi_{5}\right)\right)\right|,
\end{aligned}
$$

Fig. 5. shows the forces on the eccentric.

When determining the circumferential force on the left eccentric, the phase shift between the eccentric cranks $\phi$ should be taken into account.

$$
F_{t 4 L}\left(\varphi_{3}\right)=F_{t 4 R}\left(\varphi_{3}+\phi\right)
$$




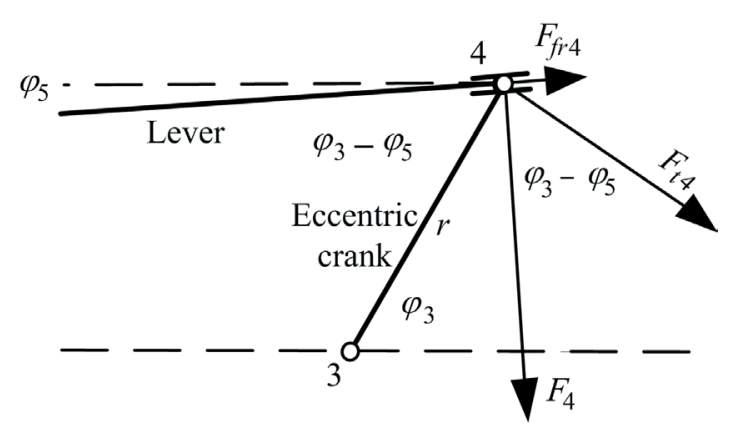

Fig. 5. Forces on the eccentric crank

\section{ANALYSIS OF FRICTION LOSSES IN THE ECCENTRIC SLIDER}

During tests, it has been shown that the main source of friction losses was slider of the eccentric, so we will pay special attention to these losses in this paper.

\subsection{Shape of the Slider}

In the initial phase of the test rig development, we evaluated two concepts of the transfer of force from the eccentric to the lever. In the first concept, the force is transmitted from the eccentric to the lever via an intermediate connecting rod, and in the second concept it is transmitted directly via the slider. The springs are not loaded under the same conditions in the first concept, so the concept with a slider was selected.

Regarding the type of friction, a rolling or sliding design of the slider is possible. The force is transmitted via rolling elements in the rolling design. The force of friction is small, friction losses are minimal, and heating of elements is negligible. Simplified implementation of the rolling version is presented in Fig. 7a. Pushing the lever is carried out with a roller, which rolls on the rails in the groove of the lever.
The rail and roller wear, so the distance between the rails is continuously adjustable. During the tests, we monitored the wear of the guides and roller. At frequencies up to $2 \mathrm{~Hz}$, the wear of the roller and guide was within acceptable limits. At higher frequencies, we noticed uneven wear along the guide. Maximum wear occurred in the outermost roller positions, while we did not notice wear in the region of the largest rolling pressure. Roller kinematics was recorded with a fast camera. We found that increased wear was caused by the roller slip in extreme positions. Wear of the rail is presented in Fig. 6.

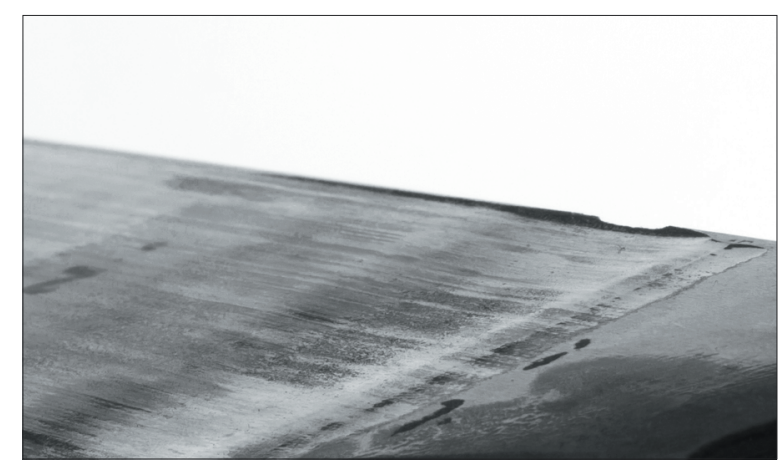

Fig. 6. Wear of the rail

Because of uneven wear of the rail and the need for frequent replacement of rails, we switched to the sliding design. We used a square block with replaceable sliding pads (Fig. 7b) instead of a roller. The test rig is currently operating with a sliding version in spite of increased friction force and the increased thermal load of elements. In the next step, we will calculate the friction power on the slider, which is critical for the wear and heat load of sliding pads.
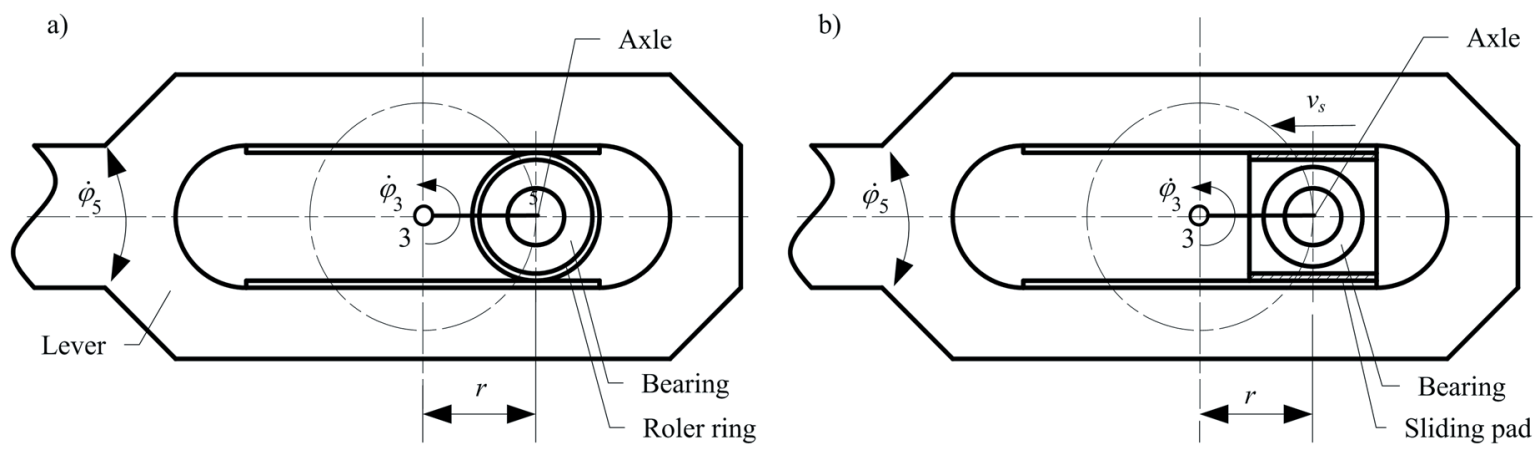

Fig. 7. Structural designs of slider 


\subsection{Friction Power on the Slider}

The friction power on the slider depends on the force of friction and sliding velocity. During rotation of the eccentric crank, both the friction force and sliding velocity vary. The maximum sliding velocity is equal to the peripheral velocity of the eccentric. At the frequency of $3.5 \mathrm{~Hz}$ and given test rig setup ( $R=0.9 \mathrm{~m}, r=0.095 \mathrm{~m})$, the maximum sliding velocity is $v_{s 4 \max }=2.1 \mathrm{~m} / \mathrm{s}$.

$$
\begin{gathered}
P_{f r 4}\left(\varphi_{3}\right)=F_{f r 4} \cdot v_{s 4}=F_{4}\left(\varphi_{3}\right) \cdot \mu \cdot v_{s 4}, \\
P_{f r 4}\left(\varphi_{3}\right)=M_{f r 4} \cdot \dot{\varphi}_{3}= \\
=F_{4}\left(\varphi_{3}\right) \cdot \mu \cdot \cos \left(\frac{\pi}{2}-\left(\varphi_{3}-\varphi_{5}\right)\right) \cdot r \cdot \dot{\varphi}_{3} .
\end{gathered}
$$

Fig. 8 shows the power curve if only the working forces of springs are taken into account, without considering the weight of the lever and inertia force. The power curve peaks appear at the extreme positions of the lever. The medium friction power is $0.75 \mathrm{~kW}$. The actual friction power on the slider is shown in Fig. 9. Due to the inertia force, the normal force on the slider is reduced, and so is the frictional force. The average friction power is thus lower: $0.4 \mathrm{~kW}$. The upper side of the slider is more loaded than the lower side due to the weight of the lever, resulting in two different power peaks. Higher peaks correspond to the friction power on the upper slider pad and lower peaks correspond to the situation on the lower pad. By integrating the power curve, the friction work is obtained, which is equal to the heat on the sliding surfaces.

\section{ANALYSIS OF FRICTION LOSSES IN BEARINGS}

\subsection{Friction Losses in the Lever Pivot}

Rolling or sliding bearings can be used for the pivot axle. A version with rolling bearings is more favourable in terms of losses, but is less favourable in terms of maintenance costs. Small angles of rotation (in our case $\varphi_{5 \max }= \pm 3.4^{\circ}$ ) cause grooves in bearing rings under rolling elements (Fig. 10); the play of the bearings increases and after about three months of operation it is necessary to replace the bearings. The average power of friction is only $3 \mathrm{~W}$ and is negligible in comparison with other losses.

\subsection{Friction Losses in Shaft Bearings}

We took into account constant radial forces on pulleys when we calculated the losses in bearings on Shafts 1 and 2, and we considered forces on both eccentrics and on the pulley for bearings on Shaft 3 .

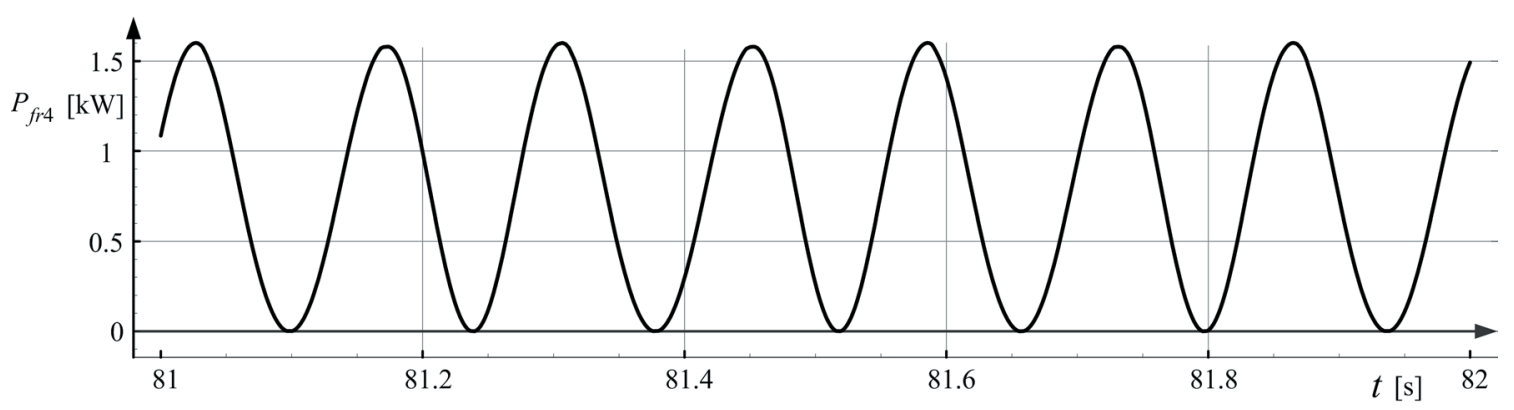

Fig. 8. Friction power on the slider (only working forces of the spring taken into account, $\mu=0.05$ )

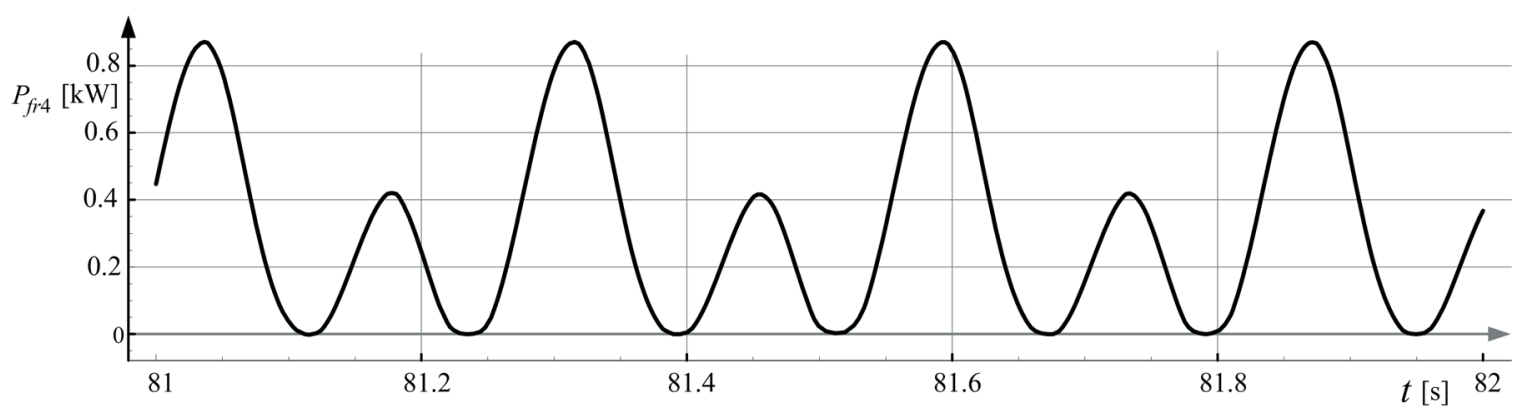

Fig. 9. Friction power on the slider (inertia and mass of the lever taken into account) 


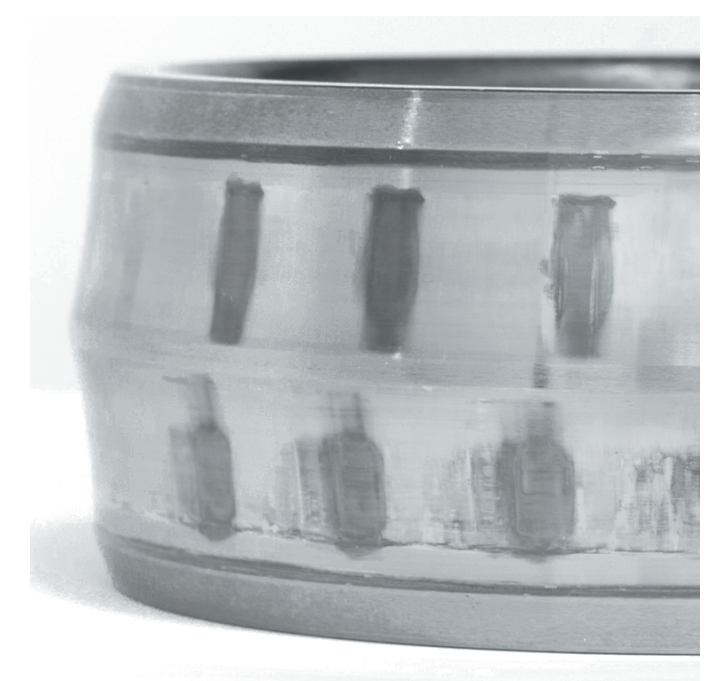

Fig. 10. Damaged bearing of lever pivot

Data on the coefficient of friction were obtained from the manufacturers of bearings. We used the coefficient of friction $\mu_{b}=0.0018$ for the selected bearings. The bearings are labelled with capital letters from $\mathbf{A}$ to $\mathbf{F}$ on Fig. 3. Radial load was calculated for individual bearings. The results of the calculation are presented in Table 1. Radial forces on bearings $\mathbf{E}$ and $\mathbf{F}$ change during rotation. We used medium values of forces.

$$
P_{f r, b}=M_{f r, b} \cdot \omega_{b}=F_{b} \cdot \frac{d_{b}}{2} \cdot \mu_{b} \cdot \omega_{b} .
$$

It is expected that the total losses of power on all roller bearings and seals are about $0.5 \mathrm{~kW}$.

Table 1. Energy losses in bearings

\begin{tabular}{ccccc}
\hline Bearing & $\omega_{b}\left[\mathrm{~s}^{-1}\right]$ & $F_{b}[\mathrm{~N}]$ & $d_{b}[\mathrm{~mm}]$ & $P_{f r, b}[\mathrm{~W}]$ \\
\hline $\mathbf{A}$ & 151.8 & 1990 & 60 & 16.3 \\
\hline B & 151.8 & 6400 & 60 & 52.5 \\
\hline C & 47.3 & 6450 & 80 & 22 \\
\hline $\mathbf{D}$ & 47.3 & 10200 & 80 & 34.8 \\
\hline $\mathbf{E}$ & 22 & 20000 & 90 & 35.6 \\
\hline $\mathbf{F}$ & 22 & 20000 & 90 & 35.6 \\
\hline
\end{tabular}

\section{POWER NEEDED FOR OPERATION AND START-UP}

Knowing circumferential forces on both eccentrics and friction losses, it is possible to calculate the required power for the drive.

$$
P_{1}=\left(F_{t 4 L}+F_{t 4 R}\right) \cdot r \cdot \dot{\varphi}_{3}+P_{f r} .
$$

The medium work for one cycle is obtained by numerical integration of the power curve.

$$
W=\int_{t_{s}}^{t_{s}+\frac{2 \pi}{\omega_{1}}} P_{1}(t) d t .
$$

The required power of the electric motor is:

$$
W=P_{1} \cdot \frac{2 \pi}{\omega_{1}} \Rightarrow P_{1}=\frac{W \cdot \omega_{1}}{2 \pi} .
$$

The power curve on the shaft 1 is shown in Fig. 11. The difference between the maximum and minimum power is $13.7 \mathrm{~kW}$. The power needed for the testing (average power) is about $3.8 \mathrm{~kW}$. Fig. 12 shows the power curve during a test of one pair of springs. The work returned by the upper spring during release is partially used for loading the lower spring. The difference between the maximum and minimum power is $18 \mathrm{~kW}$, which is $4.3 \mathrm{~kW}$ more than when testing four springs. Medium power is $4.6 \mathrm{~kW}$. When testing one spring, the spring returns the loading work (reduced by hysteresis and friction losses) to the driving system. The difference between the maximum and minimum power is $66 \mathrm{~kW}$. The selected flywheel cannot provide the speed variation within the required limits.

Energy efficiency of the new test rig is proven by the actual current flowing through the electrical motor winding. It depends on the torque load of the electric motor shaft during the test. The rated current at the rated torque is known for the selected electric motor (in our case, the rated power is $11 \mathrm{~kW}$ ). The actual current reaches about $40 \%$ of the rated current during the test of the selected spring. This means that the power of $4.4 \mathrm{~kW}$ is needed for testing four springs ( $1.1 \mathrm{~kW}$ per spring). The power on the basis of the measured current is about $0.6 \mathrm{~kW}$ higher than the calculated value. This indicates that the friction losses are somewhat higher than predicted.

When starting the test rig, the work forces of springs are negligible. Air pressure in the springs is only gradually increased when the testing frequency is achieved. The RPM of the drive shaft is being steadily increased by the frequency control of the electric motor. The power required for start-up depends on acceleration time $t_{a}$, moments of inertia $J_{1}$, angular velocity of the shaft $\omega_{1}$ and losses. Friction losses increase with rotational speed and are the highest at the end of the start-up.

$$
P_{1 a}(t)=J_{1} \cdot \frac{\omega_{1 n}}{t_{a}} \cdot \omega_{1}(t)+P_{f r}(t) .
$$




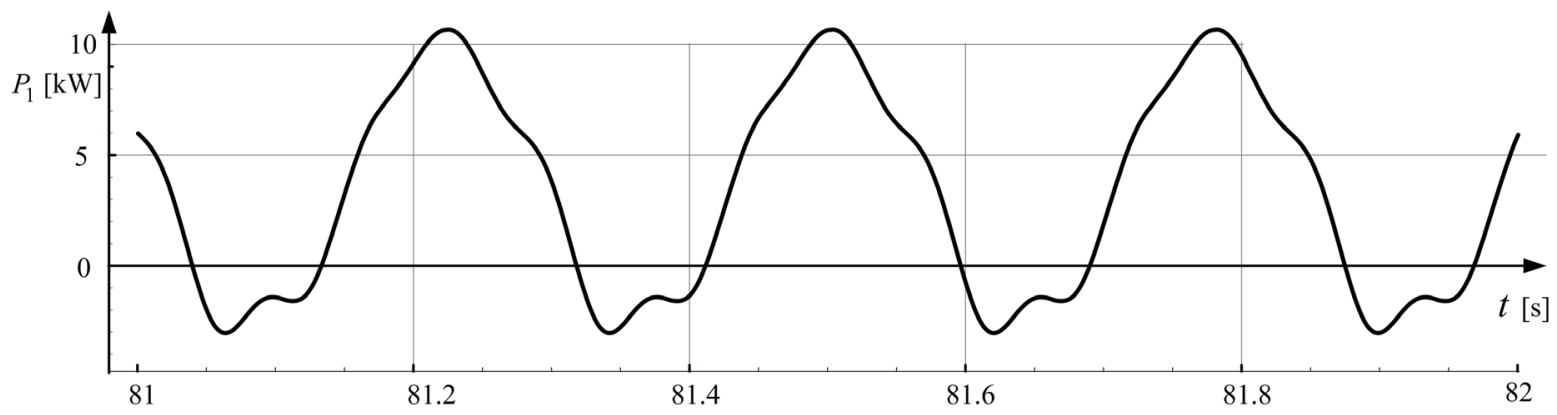

Fig. 11. Power on Shaft 1 (four springs, $R=900 \mathrm{~mm}, \mu=0.05$ )

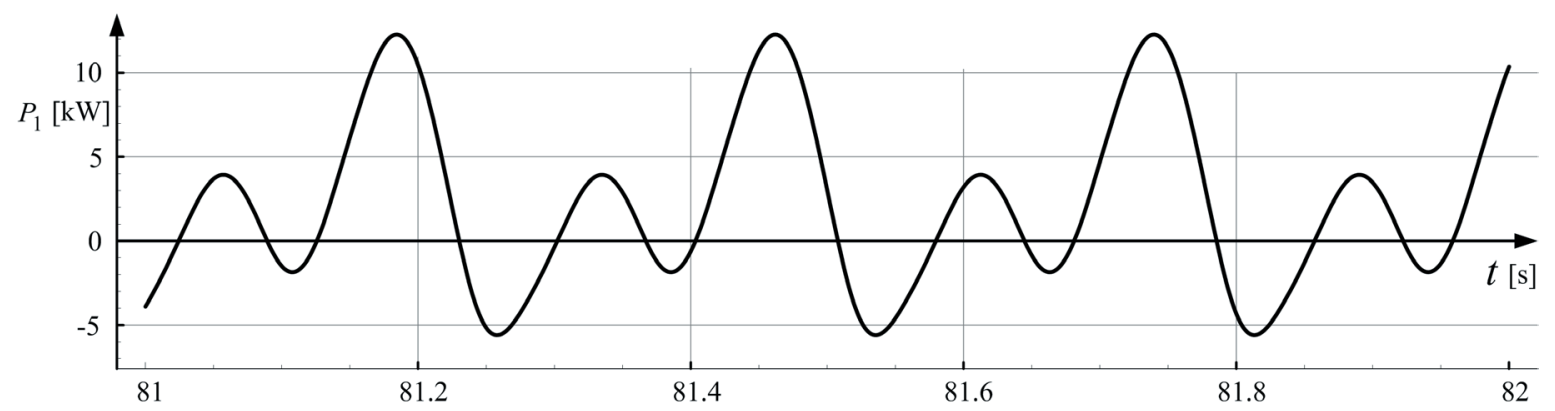

Fig. 12. Power on Shaft 1 (one pair of springs)

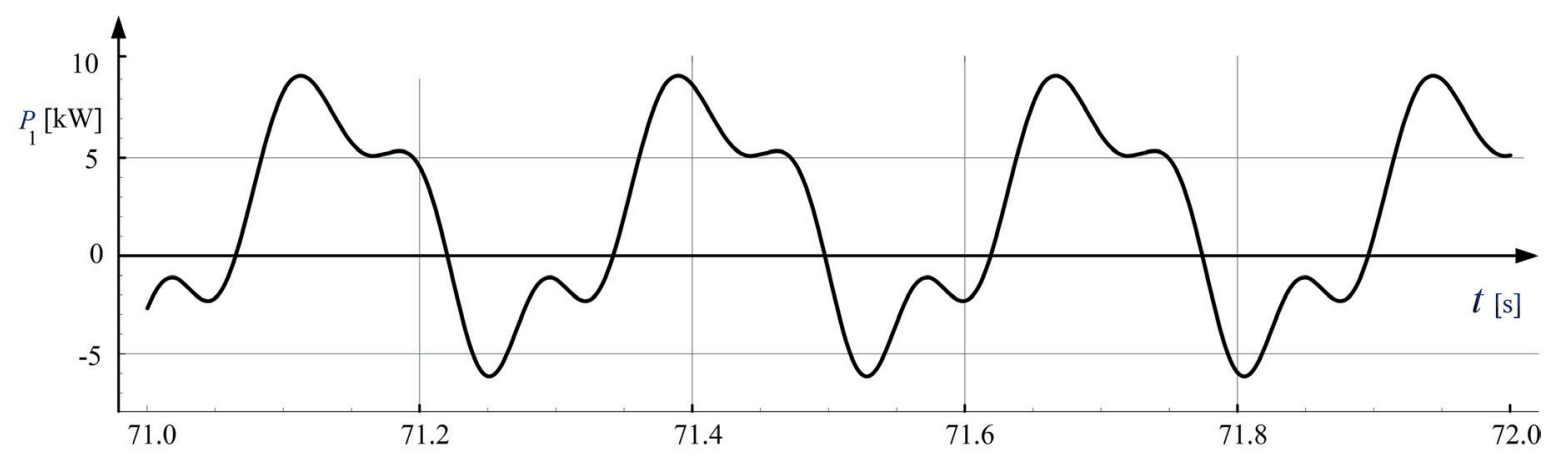

Fig. 13. Power on Shaft 1 at the end of acceleration (behind the flywheel)

Start-up power increases linearly as a function of rotational speed. If the start-up time is $60 \mathrm{~s}$ and the testing frequency is $3.5 \mathrm{~Hz}\left(\omega_{1 n}=153 \mathrm{~min}^{-1}\right)$, then $15.5 \mathrm{~kW}$ of power is needed at the end of startup. Fig. 13 presents the power curve on Shaft 1 after start-up. The fluctuation of power occurs due to the acceleration (deceleration) of the lever and the lifting (lowering) the lever. By gradually increasing the pressure in springs, work forces increase. When medium pressure reaches its final value, the need for power varies as shown in Fig. 11.

\section{EFFECT OF FLYWHEEL ON THE ENERGY SITUATION}

A flywheel was installed on Shaft 1, which rotates at the rotational frequency of the electric motor $\omega_{n}$.
Calculation of the moment of inertia of the flywheel was based on the equation of motion for Shaft 1 presented in [9]. The moment of the load $M_{t 1 L}$ affects the shaft behind the flywheel and moment of the drive $M_{t 1 D}$ affects the shaft before the flywheel.

$$
\begin{gathered}
M_{t 1 D}+M_{t 1 L}=J_{1} \cdot \frac{d}{d t}\left(\frac{d \varphi_{1}}{d t}\right)= \\
=J_{1} \cdot \frac{d \varphi_{1}}{d t} \cdot \frac{d}{d \varphi_{1}}\left(\frac{d \varphi_{1}}{d t}\right)=J_{1} \cdot \omega_{1} \cdot \frac{d \omega_{1}}{d \varphi_{1}} \\
\left(M_{t 1 D}+M_{t 1 L}\right) \cdot d \varphi_{1}=J_{1} \cdot \omega_{1} \cdot d \omega_{1} .
\end{gathered}
$$

By integrating both sides of the equation we obtain: 


$$
\begin{gathered}
\int_{\varphi_{1,1}}^{\varphi_{1,2}}\left(M_{t 1 D}+M_{t 1 L}\right) \cdot d \varphi_{1}=\int_{\omega_{1,1}}^{\omega_{1,2}} J_{1} \cdot \omega_{1} \cdot d \omega_{1}= \\
=\frac{1}{2} \cdot J_{1} \cdot\left(\omega_{1,2}^{2}-\omega_{1,1}^{2}\right) .
\end{gathered}
$$

The left side of the equation represents the work, and the right side represents the change in kinetic energy of the flywheel. We are interested in the largest change within a single cycle.

$$
\begin{gathered}
\Delta E_{\max }=\frac{1}{2} \cdot J_{1} \cdot\left(\omega_{1 \max }^{2}-\omega_{1 \min }^{2}\right)= \\
=\frac{1}{2} \cdot J_{1} \cdot\left(\omega_{1 \max }-\omega_{1 \min }\right) \cdot\left(\omega_{1 \max }+\omega_{1 \min }\right) .
\end{gathered}
$$

With the introduction of the medium angular velocity:

$$
\omega_{1 n}=\frac{\left(\omega_{1 \max }+\omega_{1 \min }\right)}{2},
$$

and coefficient of variation of velocity:

$$
C_{S}=\frac{\left(\omega_{1 \max }-\omega_{1 \min }\right)}{\omega_{1 n}},
$$

the equation takes the form:

$$
\Delta E_{\max }=J_{1} \cdot \omega_{1 n}^{2} \cdot C_{S} .
$$

The difference between the largest surplus and shortage of power with respect to the medium work for one cycle (one revolution of the eccentric) and thus the maximum change in kinetic energy of the flywheel is obtained from the power diagram (Fig. 11). The degree of non-uniformity in our case is:

$$
C_{S}=\frac{\Delta E_{\max }}{J_{1} \cdot \omega_{1 n}^{2}}=\frac{1120}{35 \cdot 153^{2}}=0.0054
$$

Variation of rotational speed is minimal. Results have also been confirmed by measuring the rotational speeds on the test rig. Identical results have been achieved by solving the differential equation of the drive in [10].

\section{CONCLUSIONS}

Tests of endurance of air springs require significant amounts of time. The test rig discussed in this paper operates seven days a week, 24 hours a day. Testing costs were an important criterion that had to be considered during the design process. The costs consist of the costs of building the test rig, maintenance costs and energy costs. The test rig allows the use of work returned by the spring during unloading. In this way, we achieved a significant reduction of energy costs and simultaneously increased the number of tests.

The energy conditions change by changing the geometrical settings and load frequency on the test rig. We developed a computer program to calculate the power and work. The geometrical settings of the test rig, measured spring characteristics, and test frequency are entered into the program. The program allows us to quickly assess the risk of thermal overload of critical elements and to calculate the actual energy losses. The power required for testing is calculated by taking into account the working forces of a spring, inertia forces, spring hysteresis and friction losses. The analyses prove that the output energy in a single air spring can with only very limited loses be transferred into the input energy of other three air springs.

Energy efficiency of the new test rig is proven by the mesured current flowing through the electrical motor winding. The actual current reaches $40 \%$ of the rated current during the test of the selected spring. This means that the power of about 4.4 $\mathrm{kW}$ is needed for testing four springs $(1.1 \mathrm{~kW}$ per spring). A hydraulic test rig and mechanical test rig for simultaneous testing of two springs also operate in the laboratory of the manufacturer, for which we developed the new test rig. Approximately $30 \mathrm{~kW}$ (maximal) is required in hydraulic test rig for loading one spring with a frequency of $3.5 \mathrm{~Hz} .5 .5 \mathrm{~kW}(2.75$ $\mathrm{kW}$ per spring) is needed for testing two springs on the existing mechanical test rig.

Variations of the actual current and testing frequency are negligible (less than 1\%). This proves the effect of the flywheel on the uniformity of the drive.

On the basis of the friction power, suitability of the solution is determined with respect to the heat load. Springs that are larger than the one described, cannot be tested with a frequency above $2 \mathrm{~Hz}$ due to excessive heat load of the slider. The largest springs from the set of springs can be tested at a frequency of $3.5 \mathrm{~Hz}$ only if a rolling version of the slider is used.

\section{REFERENCES}

[1] Oman, S., Nagode, M., Fajdiga, M. (2010). Estimation of airspring life based on accelerated experiments. Materials \& Design, vol. 31, no. 8, p. 3859-3868, D0l:10.1016/j. matdes.2010.03.044.

[2] Oman, S., Nagode, M. (2013). On the influence of the cord angle on air-spring fatigue life. Engineering Failure Analysis, vol. 27, p. 61-73, DOl:10.1016/j.engfailanal.2012.09.002. 
[3] Bešter, T., Fajdiga, M., Nagode, M. (2014). Application of constant amplitude dynamic test for life prediction of air springs at various control parameters. Strojniški vestnik Journal of Mechanical Engineering, vol. 60, no. 4, p. 241-248, DOl:10.5545/sv-jme.2013.1348.

[4] Harris, C.M. (1988). Shock and Vibration Handbook-Air Suspension and Active Vibration-Isolation Systems. chap. 33, McGraw-Hill, London.

[5] Bank, T.A. (1980). Some ABC's of air spring suspensions for commercial road vehicles, SAE, SAE Technical paper 800482 , DOl: $10.4271 / 800482$.

[6] Hirtreiter, A.B. (1973). Principles and applications of pneumatic springs. SAE. SAE technical paper 730273 , DOI:10.4271/730273.

[7] Chen, Y., He, I., King, M., Chen, W., Wang, C., Zhang, W. (2013). Model development and dynamic load-sharing analysis of longitudinal-connected air suspensions. Strojniški vestnik Journal of Mechanical Engineering, vol. 59, no. 1, p. 14-24, DOl:10.5545/sv-jme.2012.755.

[8] Mankovits, T., Szabo, T., Kocsis, I., Paczelt, I. (2014). Optimisation of the shape of axi-symmetric rubber bumpers. Strojniški vestnik - Journal of Mechanical Engineering, vol. 60, no. 1, p. 61-71, Dol:10.5545/sv-jme.2013.1315.

[9] Cleghorn, W.L. (2010). Mechanics of Machines, Oxford University Press, Oxford.

[10] Okorn, I., Fajdiga, M., Nagode, M. (2010). Analysis of test rig drive for air springs. Proceedings of the Institution of Mechanical Engineers Part C: Journal of Mechanical Engineering Science, vol. 224, no. 1, p. 263-271, DOI:10.1243/09544062JMES1602. 